\title{
Community Health Worker Knowledge, Attitudes, Practices and Readiness to Manage Intimate Partner Violence
}

\author{
Zahra Saboori $^{1} \cdot$ Robert S. Gold $^{1} \cdot$ Kerry M. Green ${ }^{1} \cdot$ Min Qi Wang ${ }^{1}$ \\ Accepted: 14 June 2021 / Published online: 9 July 2021 \\ (c) The Author(s), under exclusive licence to Springer Science+Business Media, LLC, part of Springer Nature 2021
}

\begin{abstract}
Intimate partner violence (IPV) is an important public health concern with higher prevalence among women. Community health workers (CHWs) are trusted frontline public health workers that bridge gaps between communities and healthcare services. Despite their effectiveness in delivering services and improving outcomes for different chronic conditions, there is a dearth of understanding regarding CHW management of IPV. The purpose of this study is to examine knowledge, attitudes, practices, and readiness to manage IPV among a sample of CHWs $(n=152)$. Participants completed an online version of the Physician Readiness to Manage Intimate Partner Violence Survey (PREMIS), which was modified for CHW practice. Psychometrics of the newly adapted tool, along with empirical relationships between knowledge, attitudes, and readiness to manage IPV were examined. Most sub-scales yielded moderate to high reliability $(0.70<\alpha$ 's $<0.97)$, some sub-scales had low reliability $(0.57<\alpha$ 's $<0.64)$, and construct validity was established for several of the subscales. On average, many CHWs had low scores on objective knowledge of IPV (mean $=15.4$ out of 26 ), perceived preparation to manage IPV ( mean $=3.8$ out of 7), and perceived knowledge of IPV (mean $=3.7$ out of 7 ). About $56 \%$ of CHWs indicated having no previous IPV training, 34\% did not screen for IPV, and 65\% were in the contemplation stage of behavior change. Multiple regression models indicated that knowledge, staff capabilities and staff preparation were significant predictors of perceived preparedness to manage IPV (all $p$ 's $<0.05$ ). Results can inform future credentialing requirements and training programs for CHWs to better assist their clients who are victims of IPV.
\end{abstract}

Keywords Intimate partner violence $\cdot$ Community health worker $\cdot$ PREMIS, practices

\section{Introduction}

Intimate Partner Violence (IPV) is a public health problem that is more prevalent among women. As defined by the Centers for Disease Control and Prevention, IPV is "physical violence, sexual violence, stalking and/or psychological harm by a current or former intimate partner" [1]. About $25 \%$ of women and $10 \%$ of men experience physical violence, sexual violence and/or stalking while enduring an IPV-related impact during their lifetime [2].

There are many physical and mental health consequences associated with IPV. Some examples include: a history of

Zahra Saboori

zsaboori@gmail.com

1 Department of Behavioral and Community Health, University of Maryland College Park School of Public Health, 4200 Valley Drive, Suite 1234, College Park, MD 20742, USA abuse, sexually transmitted infections, unplanned pregnancies, depression, anxiety, post-traumatic stress disorder (PTSD), alcohol and substance abuse, and chronic stress [3-9]. With such diverse consequences, victims of IPV have higher rates of healthcare utilization and more interactions with healthcare providers than non-victims [7]. This puts healthcare professionals in a unique position to be able to identify clients who are victims of IPV and refer them to appropriate resources [10].

Screening and counseling patients about IPV can assist in identifying IPV, reduce the severity and frequency of violence between intimate partners, improve safety, and reduce the impact of subsequent health consequences [11-14]. The U.S. Preventive Services Task Force recommends that clinicians screen all women of childbearing age for IPV and provide referrals to IPV resources for those who screen positive [15]. Yet, Liebschutz et al. [16] found that many healthcare providers lack the skills of asking patients about IPV, and the knowledge of making referrals once IPV has 
been identified. Even though screening and identification of IPV has increased more recently among providers, many continue to not screen for/identify IPV or refer clients to services $[17,18]$.

Despite much research dedicated to physicians addressing IPV, a crucial part of the healthcare workforce has been understudied. Community health worker $(\mathrm{CHW})$ is an umbrella term that defines paraprofessionals or "frontline public health workers who [are] trusted member[s] of and/ or have... a close understanding of the communities they serve [and are] intermediaries between health/social services and the community" [19]. CHW-led interventions have been effective in increasing access to health/social services, improving chronic disease management, and enhancing prevention services [20]. To date, few studies have examined the effectiveness of CHWs in managing clients who are victims of IPV [21].

The Physician Readiness to Manage Intimate Partner Violence Survey (PREMIS) is a valid and reliable tool that has been used to evaluate physicians' knowledge, attitudes, and practice skills necessary to manage patients who are victims of IPV [22]. This tool has been modified for implementation among social workers, pharmacists, medical students, OBGYN physicians, and other healthcare professionals and has yielded promising psychometrics [23]. While there have been protocols, best practices, and tools introduced to train and evaluate physicians in addressing IPV, there are currently no best practices or applied strategies for CHWs. The purpose of this study was to examine knowledge, attitudes, practices, and preparedness to manage IPV among a sample of CHWs using a modified PREMIS for CHWs. The following research questions were addressed (1) How prepared are CHWs to manage clients who are victims of IPV? (2) How valid and reliable is the modified PREMIS for use with CHWs?

\section{Methods}

\section{Study Design and Procedures}

This cross-sectional study was conducted in two phases. During the first phase, the PREMIS was modified based on existing literature to fit $\mathrm{CHWs}$ roles and responsibilities. Items that were irrelevant to CHWs practice were removed. All verbiage containing "patients" were reworded to "clients". A panel of four experts (two CHWs with at least two years of experience and two CHW trainers/administrators), recruited through purposive sampling, reviewed the modified PREMIS survey for content, clarity, ambiguity, and appropriateness for $\mathrm{CHW}$ practice. All changes and recommendations were factored into the final version of the modified PREMIS for CHWs. The finalized instrument was pilot tested among a group of $\mathrm{PhD}$ students $(\mathrm{n}=3)$ prior to implementation in the CHW survey. Participants were recruited using snowball sampling via listservs and points of contact at different organizations employing CHWs. Recruitment took place between October 2020-December 2020. The anonymous survey was returned online via Qualtrics ${ }^{\circledR}$ by a group of practicing CHWs $(n=164)$. Of these, 12 were eliminated from the analyses due to having greater than $50 \%$ missing data. Therefore, the final data producing sample was 152 practicing CHWs. All participants who chose to enter their information in a separate survey received a 10-dollar Amazon gift card. This study was approved by Institutional Review Boards at the University and Health Department of the State under study. Permission was also obtained from the original authors, Short et al. [22], to conduct this study among CHWs.

\section{Measures}

\section{Demographic and Background Characteristics}

Participants were asked questions about their age, gender, education, CHW certification, years of experience, work setting, and activities as a CHW. Age and years of experience were continuous variables. Gender (male, female, transgender, prefer not to say) and education (ranging from high school diploma to masters and higher) were treated as categorical variables. Setting of work, and types of activities as a CHW employed a "check all that apply" response format. CHW certification was a dichotomous variable (yes/no). Background characteristics included a question that asked "Have you ever received IPV training" with a dichotomous (yes/no) response format. Participants who responded yes to the IPV training question were asked questions about the duration of their training in IPV and types of IPV training, both of which were categorical variables. Perceived preparedness to manage IPV included seven items that asked: "Please circle the number which best describes how prepared you feel to perform the following" using a 7-point Likert response format $(1=$ not prepared to $7=$ quite well prepared). A sample item was: "Make appropriate referrals for IPV". Perceived knowledge about IPV included 10 items that asked: "How much do you feel you know about the following" using a 7-point Likert response format $(1=$ nothing to $7=$ very much). A sample item was: "signs and symptoms of IPV".

\section{Objective IPV Knowledge}

This section of the modified PREMIS for CHWs included six overall questions with multiple sub-questions. The first item employed a multiple-choice categorical response format that asked participants: (1) "What is the strongest risk 
factor for being a victim of IPV"? Similarly, the second item asked: (2) "Which one of the following is generally true about perpetrators". The next three questions used a check all that apply response format and asked the following: (3) "Which of the following are warning signs that a client may have been abused by his/her partner"? (4) "Which of the following are reasons an IPV victim may not be able to leave a violent relationship"? and (5) "Which of the following are the most appropriate ways to ask about IPV"? Finally, the last general question asked participants to circle true, false or do not know for each of the 9 sub-questions. Each knowledge question or subquestion was recoded to a dichotomous variable $(0=$ incorrect, $1=$ correct). A summative knowledge score was calculated based on the number of correct responses (24 points possible).

\section{IPV Opinions}

This section asked participant 26 questions relating to opinions about CHW management of IPV using a 7-point Likert scale ranging from $1=$ strongly disagree to $7=$ strongly agree. A sample item was: "If an IPV victim does not acknowledge the abuse, there is very little I can do to help". As mentioned previously, all items that were irrelevant to CHW scope of practice were removed in this modified PREMIS for CHWs (e.g., items relating to IPV reporting requirements for clinicians or items pertaining to physical exams for IPV victims).

\section{Readiness to Change}

In order to assess each participant's readiness to adopt appropriate IPV practices into their work, a modified University of Rhode Island Change Assessment (URICA) was developed. Originally designed to address the readiness to change problematic alcoholic behaviors, the scale is a common tool that examines whether or not individuals express a willingness to change their behaviors [24]. This modified tool was used to categorize individuals into one of four different stages within the Stages of Change construct of the Transtheoretical Model [25]. These four stages include (1) Precontemplation (not thinking about changing IPV management behaviors); (2) Contemplation (evaluating pros and cons of changing IPV management behaviors); (3) Action (has made active changes to IPV management behaviors); (4) Maintenance (has changed IPV management behaviors and maintained that change for over 6 months). The URICA-12 was adapted to be consistent with behaviors relating to CHW management of IPV identified within the PREMIS and the literature. The modified URICA-12 asks 12 questions relating to CHW management of IPV that utilize a 5-point Likert scale ranging from $1=$ strongly disagree to $5=$ strongly agree. Each stage of change included three questions. As conducted in the original scale, mean scores were calculated for each contemplation, action, and maintenance items. The precontemplation mean score was subtracted from the sum of the mean contemplation, action, and maintenance score, and this yielded the final mean readiness to change score. Readiness scores below 8 indicate the precontemplation stage. Scores between 8.01 and 11.99 indicate the contemplation stage. Scores above 12-14 indicate the preparation/action stage. Final readiness scores were recoded to a categorical variable, thereby designating the appropriate stage of change for each respondent.

\section{Data Analyses}

Data analyses were conducted using The Statistical Package for Social Sciences (SPSS) Version 27 [26]. All respondent surveys with greater than $50 \%$ missing data were removed from analyses. Patterns of missing data were examined by recoding demographic variables to dichotomous variables (missing vs. non-missing) to examine whether there were any significant differences between the variables. Insignificant relationships were examined when conducting t- tests between variables comparing missing and non-missing values, thereby suggesting data missing completely at random (MCAR). Multiple imputation was conducted for each section of the survey that had greater than 5\% missing data. Demographic variables (age, gender, years worked as a CHW, education, and certification) were used as predictors to impute each of the main study variables using five imputations and an average value for imputed data. Imputed variables included (1) opinions (5-8\% missing); (2) readiness to change (8-9\% missing); (3) years worked as a CHW (17.8\% missing); IPV practices (11-13\% missing). Missing values for objective knowledge (1.3\%), perceived knowledge $(0 \%)$, and perceived preparation $(0 \%)$ were not imputed due to having low to negligible missing data. Because changes were made to the instrument to reflect $\mathrm{CHW}$ practice, psychometrics of the modified PREMIS for CHWs were examined. To address the second research question, principal components analysis with varimax rotation was conducted to examine underlying factor structures for the newly modified opinion scales and readiness to change variables. All negatively worded items were reverse coded. A factor loading of 0.4 was used as the criterion for an item to become a part of a scale. 
A mean score was calculated for each emergent opinion subscale. A mean readiness to change score was also calculated (described above). Reliability of the modified tool was examined by calculating Cronbach's alphas for each opinion subscale, readiness to change subscale, perceived preparedness to manage IPV, and perceived IPV knowledge. Kuder-Richardson formula 20 was used to assess the reliability of the objective knowledge scales [27]. Pearson's correlations were also calculated between each subscale to determine construct validity. The following ranges were used to interpret correlation coefficients: (1) Negligible (0.0-0.3), (2) Low (0.31-0.5), (3) Moderate (0.51-0.7), or (4) High (0.71-0.9) [28].

For the first research question, univariate descriptive statistics were calculated for categorical and continuous variables. Multiple linear regression models were also examined with knowledge and opinion sub-scales as predictors and preparedness to manage IPV as the outcome. IPV training, $\mathrm{CHW}$ certification, and years worked as a $\mathrm{CHW}$ were treated as covariates.

\section{Results}

\section{Demographics and Background Characteristics of Participants}

The demographics and background characteristics of participants $(n=152)$ are included in Table 1 . The mean age of participants was 43.7 years $(\mathrm{SD}=12.4)$. On average, participants worked in their $\mathrm{CHW}$ positions for 6.2 years $(\mathrm{SD}=5.9)$. Most participants were female $(88.2 \%)$, held a community health worker title $(78.9 \%)$, and were certified CHWs (78.3\%). With regards to education level, about $25.7 \%$ of participants had some college, $31.6 \%$ had an associate's degree, and $15.8 \%$ had a master's or higher. Most CHWs worked in hospital/clinic settings (43.4\%), and some worked in community-based organizations (39.5\%). Activities that CHWs reported included health education $(85.5 \%)$, resource referrals $(81.6 \%)$, advocacy $(79.6 \%)$, home visitations $(75.0 \%)$, patient navigation $(67.1 \%)$, program enrollment $(61.8 \%)$ and case management (59.2\%). Most participants reported being in the contemplation stage of change $(64.5 \%)$

The majority of participants reported having received no IPV training (55.9\%). Of those who had previous IPV training, about $35 \%$ had received more than one hour and less than one day, $32.8 \%$ had received 1-2 days of training, and $25 \%$ had received more than three days of training (Table 2). Most participants who received training attended a lecture or talk (62.6\%) and/or a skills-based workshop.

\section{Practice Issues}

Since there are currently no established standards of practice for dealing with IPV among CHWs, Table 3 illustrates practice issues of IPV management as reported by the responding CHWs. Less than half of individuals reported seeing 1-5 cases of IPV within the past 6 months (44.7\%). Some participants reported not being in clinical practice $(31.6 \%)$, not screening for IPV (34.2\%), or not having training to screen for IPV (31.6\%). About half of CHWs reported not being familiar with their institution's policies on IPV (46.7\%), and $19.7 \%$ reported that IPV was not applicable to their practice. When asked what actions they have taken when identifying IPV in the past 6 months, about two thirds of participants $(61.8 \%)$ reported not having identified IPV. More than one third of participants were unsure of their workplace's protocol for dealing with IPV (34.9\%). More than half of participants indicated that they did not have adequate knowledge about IPV referral resources $(52.6 \%)$ and $13.2 \%$ indicated that this was not applicable to their client population. Less than half of participants indicated that IPV education materials were available at their practice $(48.0 \%)$.

\section{Psychometrics of Modified PREMIS for CHWS}

Table 4 highlights average scores, definitions, and reliability for all PREMIS scales and opinion sub-scales. The principal components analysis of opinion questions yielded a 6-factor solution, with sub-scales having low to high reliability. The factors included (1) Staff Preparation ( $\alpha=0.88)$; (2) Staff Response ( $\alpha=0.74)$; (3) Staff Constraints $(\alpha=0.70)$; (4) Staff Capabilities $(\alpha=0.60)$; (5) Alcohol/Drugs $(\alpha=0.57)$; (6) Victim Understanding $(\alpha=0.64)$. All items with factor loadings below 0.40 were removed from the final analyses. A seventh factor comprised of three items yielded a Cronbach's alpha of 0.39 and was removed from the final analysis. The average score of staff preparation (training or skills to address IPV) was lowest of all opinion sub-scales (Mean =4.2, $\mathrm{SD}=1.57$ ), and the average score of staff constraints (factors that make it difficult to manage IPV) was highest among all subscales $($ Mean $=5.8, \mathrm{SD}=1.23)$. The average score of participants on the perceived preparation to manage IPV scale was $3.8(\mathrm{SD}=1.37)$, and perceived knowledge was 3.7 $(\mathrm{SD}=1.37)$. The maximum possible score for most scales and sub-scales was a 7 . The average objective knowledge score was $15.4(\mathrm{SD}=4.0)$, with a maximum score of 19 and a minimum score of 2 . The maximum possible score on the knowledge section was 26.

Most opinion sub-scales were similar to that of the original PREMIS for physicians, but since certain items 
Table 1 Participant characteristics

\begin{tabular}{|c|c|}
\hline Variable & $\begin{array}{l}N=152 \\
n(\%)\end{array}$ \\
\hline Age & $43.7(12.4)^{\mathrm{a}}$ \\
\hline Years worked as a $\mathrm{CHW}$ & $6.2(5.9)^{\mathrm{a}}$ \\
\hline \multicolumn{2}{|l|}{ Gender } \\
\hline Male & $15(9.9)$ \\
\hline Female & $134(88.2)$ \\
\hline Transgender & $2(1.3)$ \\
\hline \multicolumn{2}{|l|}{ CHW titles } \\
\hline Promotora de salud & $3(2.0)$ \\
\hline Patient navigator & $8(5.3)$ \\
\hline Community health worker & $120(78.9)$ \\
\hline Health educator & $25(16.4)$ \\
\hline Outreach worker & $32(21.1)$ \\
\hline Care coordinator & $19(12.5)$ \\
\hline \multicolumn{2}{|l|}{ Highest level of education } \\
\hline Less than high school/GED & $3(20)$ \\
\hline High school diploma/GED & $20(13.2)$ \\
\hline Post high school training & $4(2.6)$ \\
\hline Some college & $39(25.7)$ \\
\hline Associates degree & $48(31.6)$ \\
\hline Bachelor's degree & $14(9.2)$ \\
\hline Masters or higher & $24(15.8)$ \\
\hline \multicolumn{2}{|l|}{ Readiness to change } \\
\hline Precontemplation & $47(30.9)$ \\
\hline Contemplation & $98(64.5)$ \\
\hline Preparation/action & $7(4.6)$ \\
\hline \multicolumn{2}{|l|}{ CHW certification } \\
\hline Yes & $119(78.3)$ \\
\hline No & $32(21.1)$ \\
\hline \multicolumn{2}{|l|}{ CHW activities } \\
\hline Home visitations & $114(75.0)$ \\
\hline Patient navigation & $102(67.1)$ \\
\hline Environmental scans & 28 (18.4) \\
\hline Advocacy & $121(79.6)$ \\
\hline Health education & $125(85.5)$ \\
\hline Direct service & $21(13.8)$ \\
\hline Resource referral & 124 (81.6) \\
\hline Case management & $90(59.2)$ \\
\hline Screening or testing & $41(27.0)$ \\
\hline Program enrollment & $94(61.8)$ \\
\hline \multicolumn{2}{|l|}{ Ever received IPV training } \\
\hline Yes & $67(44.1)$ \\
\hline No & $85(55.9)$ \\
\hline \multicolumn{2}{|l|}{ Work setting } \\
\hline Hospital/clinic & $66(43.4)$ \\
\hline Health department & $25(16.4)$ \\
\hline School & $2(1.3)$ \\
\hline Outreach/field work & 47 (30.9) \\
\hline Community-based organization & $60(39.5)$ \\
\hline Other & $14(9.2)$ \\
\hline
\end{tabular}

${ }^{\mathrm{a}}$ Mean, standard deviation 
Table 2 Amount and types of training received among CHWs with prior IPV training

\begin{tabular}{lc}
\hline & $\begin{array}{l}N=67 \\
n(\%)\end{array}$ \\
\hline Amount of IPV training & $4(6.0)$ \\
Less than one hour & $24(35.8)$ \\
More than one hour less than one day & $22(32.8)$ \\
1-2 days & $17(25.3)$ \\
More than three days & \\
Types of training & $32(47.8)$ \\
Attended specialty training & $33(49.2)$ \\
Attended skills-based workshop & $30(44.8)$ \\
Watched video & $42(62.6)$ \\
Attended lecture or talk & $27(40.3)$ \\
Attended classroom or clinic training &
\end{tabular}

This includes all individuals who indicated they had received prior IPV training

were removed or modified due to being irrelevant to CHW scope of practice, there were some minor differences. Of opinion sub-scales, alcohol/drugs and victim autonomy were identical to the original study in terms of factor structure and similar in reliability. Staff preparation, staff constraints, and victim understanding were similar to the original authors in factor structure but varied by 1-2 items. Finally, staff response and staff capabilities were new factors that emerged as a result of the current analyses.

As completed in the original study [22] construct validity was examined by calculating Pearson's correlations between opinion sub-scales and other study variables (Table 5). The rationale was to establish whether there were significant relationships between opinion sub-scales and variables that were expected to be related. There was a high significant correlation between perceived preparation and perceived knowledge $(r=0.768, p<0.01)$, a low significant correlation between perceived knowledge and objective knowledge $(\mathrm{r}=0.345, \mathrm{p}<0.01)$, and a negligible but significant correlation between perceived preparation and objective knowledge $(\mathrm{r}=.286, \mathrm{p}<0.01)$. Staff preparation was moderately and significantly correlated with perceived preparation $(\mathrm{r}=0.522, p<0.01)$ and perceived knowledge $(r=0.647, p<0.01)$. Staff capabilities displayed moderate and significant correlations with perceived knowledge $(r=0.524, p<0.01)$, and low but significant correlations with perceived preparation $(\mathrm{r}=0.463$, $p<0.01)$. Staff response also had low and significant correlations with perceived preparation $(\mathrm{r}=0.356, p<0.01)$. There was also a low and significant correlation between victim understanding and objective knowledge $(r=0.414$, $p<0.01)$. All other correlations were either insignificant or negligible. Pearson's correlations between the readiness to change scale and the preparedness to manage IPV scales were insignificant $(r=0.026, p>0.05)$. Table 6 illustrates results of multiple regression models examining the relationships between knowledge opinion subscales, with preparedness to manage IPV as an outcome variable, while controlling for IPV training, years worked as a $\mathrm{CHW}$, and $\mathrm{CHW}$ certification. The overall model was significant $(\mathrm{F}(10,140)=20.37, p<0.001)$ with an adjusted $\mathrm{R}^{2}=0.5$. The Kaiser-Meyer-Olkin Test statistic was 0.73, which indicates middling sampling adequacy [29]. Staff preparation, and staff capabilities were statistically significant predictors of perceived preparedness to manage IPV. Knowledge and victim understanding were also marginally significant predictors of preparedness to manage IPV. Holding all variables constant, our model indicates that every 1 unit increase in staff preparation, and staff capabilities results in increased perceived preparedness to manage IPV by 0.17 staff preparation score units, and 0.38 staff capabilities score units respectively. All assumptions for linear regression were satisfied (normality, collinearity, and homoscedacity). 
Table 3 Practice Issues by CHWs Managing IPV

$N=152$

$n(\%)$

New cases of IPV in the past 6 months

None

66 (44.7)

$1-5$

44 (28.9)

$6-10$

$6(3.9)$

11-20

$11(7.2)$

21 or more

7 (4.6)

Not applicable

$16(10.5)$

Situations currently screening for IPV

Not in clinical practice

48 (31.6)

Do not screen for IPV

52 (34.2)

Do not have training to screen for IPV

48 (31.6)

Screen all new clients

39 (25.7)

Screen all new female clients

Clients with IPV indicators

19 (11.8)

Female clients during home visits

Pregnant women-specific times of pregnancy

13 (8.6)

All clients periodically

$23(15.1)$

All female clients periodically

Client referrals when identifying IPV

Therapy

40 (26.3)

Social worker/advocate

59 (38.8)

Battered women's program/shelter

57 (36.8)

National Domestic Violence/IPV home

$41(27.0)$

Police, Sherriff, or other law enforcement

33 (21.7)

Housing, educational, job/ financial assistance

34 (22.4)

Child protective services

27 (17.8)

Support group

28 (18.4)

Are you familiar with your institution's policies on screening and management of IPV?

Yes

$51(33.6)$

No

$71(46.7)$

Not applicable

30 (19.7)

Actions taken when identified IPV in the past 6 months

Have not identified IPV /No Action

$94(61.8)$

Provide information to client

49 (32.2)

Counseled client about options she/he may have

$40(26.3)$

Conducted safety assessment for victim

$24(15.8)$

Conducted safety assessment for victim's children

$15(9.9)$

Helped develop safety plan

20 (13.2)

Does your workplace have protocol- dealing with IPV?

Yes, and widely used

31(20.4)

Yes, and used to some extent

$23(15.1)$

Yes, but not used

$6(3.9)$

No

$18(13.2)$

Unsure

53 (34.9)

Not applicable to my client population

13 (8.6)

Adequate knowledge about IPV referral resources

Yes

$52(34.2)$

No

$62(40.8)$

Don't know

18 (11.8)

Not applicable to my client population

$20(13.2)$

IPV education materials available at worksite

Yes

73 (48.0)

No

52 (34.2)

Not applicable 
Table 4 CHW PREMIS scale reliability and descriptive statistics $(\mathrm{N}=152)$

\begin{tabular}{|c|c|c|c|c|}
\hline Scale & Definition & $\begin{array}{l}\text { Number } \\
\text { of Items }\end{array}$ & Mean, (SD) & Alpha \\
\hline Perceived preparation & $\begin{array}{l}\text { Mean score of items with a 7-point Likert scale measuring perceptions of CHWs } \\
\text { towards being prepared to manage IPV }\end{array}$ & 7 & $3.8(1.37)$ & 0.91 \\
\hline Perceived knowledge & $\begin{array}{l}\text { Mean score of items with a 7-point Likert scale measuring perceptions of CHWs } \\
\text { towards being knowledgeable about IPV management }\end{array}$ & 10 & $3.7(1.58)$ & 0.97 \\
\hline Objective knowledge & $\begin{array}{l}\text { Summative score of bivariate, check all that apply, and multiple-choice questions meas- } \\
\text { uring specific facts relating to IPV }\end{array}$ & 24 & $15.4(4.0)$ & 0.64 \\
\hline Staff preparation & Mean score of opinion items relating to skills or training to address or discuss IPV & 4 & $4.2(1.57)$ & 0.88 \\
\hline Staff response & Mean score of opinion items relating to asking about and responding to IPV & 3 & $4.5(1.51)$ & 0.74 \\
\hline Staff constraints & Mean score of opinion items relating to factors that make it difficult to manage IPV & 3 & $5.8(1.23)$ & 0.70 \\
\hline Staff capabilities & Mean score of opinion items about staff abilities to identify and address IPV & 3 & $4.4(1.13)$ & 0.60 \\
\hline Alcohol/drugs & Mean score of opinion items relating to alcohol or drug use & 3 & $4.3(0.93)$ & 0.57 \\
\hline Victim understanding & Mean score of opinion items relating to victims acknowledging abuse & 3 & $4.9(1.26)$ & 0.64 \\
\hline
\end{tabular}

Cronbach's alpha rounded to hundredths

Scales with 7-point Likert response format

Table 5 CHW PREMIS validity correlations between opinion subscales, perceived preparation, perceived knowledge, and objective knowledge scales $(\mathrm{N}=152)$

\begin{tabular}{lclc}
\hline Opinion subscale & $\begin{array}{l}\text { Perceived } \\
\text { preparation }\end{array}$ & $\begin{array}{l}\text { Perceived } \\
\text { knowledge }\end{array}$ & $\begin{array}{c}\text { Objective } \\
\text { knowledge }\end{array}$ \\
\hline Staff preparation & $0.522^{* *}$ & $0.647^{* *}$ & 0.158 \\
Staff capabilities & $0.463^{* *}$ & $0.524^{* *}$ & -0.048 \\
Staff response & $0.356^{* *}$ & $0.400^{* *}$ & $0.160^{*}$ \\
Staff constraints & 0.067 & $0.233^{*}$ & $0.181^{*}$ \\
Victim understanding & -0.055 & 0.101 & $0.414^{* *}$ \\
Alcohol and drugs & 0.123 & 0.113 & 0.140 \\
Perceived preparation & & $0.768^{* *}$ & $0.286^{* *}$ \\
Perceived knowledge & & & $0.345^{* *}$ \\
\hline
\end{tabular}

*Significant at the 0.05 level (2-tailed)

**Significant at the 0.01 level (2-tailed)

\section{Discussion}

One of the developmental Healthy People 2020 objectives that still remain in Healthy People 2030 is to reduce IPV across the lifespan [30]. While there is no baseline data for this objective, addressing IPV is a priority at the national level. The Health Resources and Services Administration (HRSA) recommends that the most important priority is to "train the Nation's healthcare and public health workforce to address IPV at the community and health systems levels" [31]. In this study, the original PREMIS for physicians was modified to become more appropriate for utilization among practicing CHWs. To the best of our knowledge, this study was the first of its kind that sought to examine the knowledge, attitudes, practices, and preparedness of a key segment of the healthcare workforce (CHWs) in managing a high
Table 6 Multiple regression analyses with knowledge and opinion subscales (independent variable) and perceived preparedness to manage IPV (dependent variable) $(\mathrm{N}=152)$

\begin{tabular}{lcccccc}
\hline & Unstandardized $\beta$ & SE & Standardized $\beta$ & $t$ & $p$ & Model \\
\hline Knowledge score & 0.07 & 0.02 & 0.20 & 3.02 & 0.09 & $\mathrm{~F}(10,140)=20.37$ \\
Staff preparation & 0.17 & 0.06 & 0.20 & 2.74 & $<0.001 \quad p<0.0001 \mathrm{R}^{2}=0.59$ \\
Staff response & 0.01 & 0.06 & 0.01 & 0.16 & 0.87 & Adjusted $\mathrm{R}^{2}=0.50$ \\
Staff constraints & -0.02 & 0.08 & -0.02 & -0.29 & 0.78 & \\
Staff capabilities & 0.38 & 0.08 & 0.32 & 4.73 & $<0.001$ & \\
Alcohol drugs & -0.20 & 0.08 & -0.04 & -0.64 & 0.52 & \\
Victim understanding & -0.12 & 0.08 & -0.10 & -1.69 & 0.09 & \\
\hline
\end{tabular}

Controlled for IPV training, CHW certification, and years of service as a CHW 
priority public health concern (IPV) using an evaluation tool (PREMIS) that has been validated among other populations.

Most CHWs reported engaging in home visitations, patient navigation, advocacy, health education, and resource referrals as part of their daily activities. This puts CHWs in a unique position of interacting with clients in different settings, particularly where IPV may be taking place. One feasibility study of a CHW outreach program for survivors of IPV noted that participants who received direct care and advocacy from CHWs remained actively involved in seeking services to address IPV [32]. Even though almost $80 \%$ of CHWs in our study were certified, over half of participants had not received IPV training. That being said, about two thirds of participants were in the contemplation stage of change, thereby indicating their readiness to evaluate the pros and cons of changing their IPV management behaviors.

The average knowledge score of CHWs was not high (15.4 with a maximum of 24). As an example, only $12.5 \%$ of participants knew the greatest risk factor for IPV. Perceived preparation to manage IPV (3.8 with a maximum of 7 ) and perceived knowledge (mean of 3.7 with a maximum of 7) scores were also low. In their systematic review of qualitative and quantitative studies on CHWs and paraprofessionals delivering services for survivors of sexual violence, Gatuguta et al. [21] noted that while some studies introduce different services provided by CHWs, there is a gap in understanding where CHW knowledge and skills can be utilized. Based on the most recent reports, many states are undergoing credentialing changes for CHWs and IPV training is typically not a credentialing requirement [33]. Taken together, the results suggest that although CHWs may not be very knowledgeable or well prepared to manage clients who are victims of IPV, their readiness to change their IPV management behaviors introduces the importance of considering IPV training as part of CHW credentialing.

In terms of the first research question, our results indicate that when holding other variables constant, staff capabilities (staff abilities to identify and address IPV), and staff preparation (how much skills and training CHWs had) were significant predictors for preparedness to manage IPV. Objective knowledge (how well CHWs were aware of facts about IPV) and victim understanding (how well CHWs understood victim viewpoints) were marginally significant predictors of perceived preparation to manage IPV. While the mean preparedness scores of CHWs were somewhat low, the aforementioned variables influence how well prepared CHWs are in managing IPV. This further establishes the need to explore additional opportunities for training and credentialing CHWs in IPV management. To address the second research question, similar to the original study [22] the psychometrics of the modified PREMIS for CHWs are somewhat reliable and valid. Some opinion sub-scales were consistent with the original authors in terms of factor structure and reliability. Despite this consistency, low reliability scores on certain sub-scales require that corresponding items be re-examined/modified for future use of this instrument. The newly established readiness to change scale was not correlated with perceived preparation to manage IPV, thereby suggesting the measurement of different constructs.

Despite the many strengths of this study, the results must be interpreted while considering certain limitations. Due to the cross-sectional nature of the study, it is difficult to establish temporal associations and causality. However, findings can inform future interventions, credentialing, and training opportunities regarding CHW management of IPV. This study took place among a sample of CHWs across three mid-Atlantic regional States, all of which have different credentialing requirements for CHWs. In addition, given that this survey was administered online due to COVID19 research restrictions, CHWs in remote areas or without internet access may have not been able to participate in the study. Therefore, results of this study may not be generalized to CHWs across the United States. The large number of variables in the regression model resulted in a middling sampling adequacy $(\mathrm{KMO}=0.73)$. This small subjects to variables ratio (15:1) ultimately explains the discrepancy between the $\mathrm{R}^{2}(0.59)$ and adjusted $\mathrm{R}^{2}(0.50)$. Moreover, while many scales did not display high internal consistency, the emerging psychometric results are somewhat consistent with the original validation of the PREMIS [22]. Similarly, the coding strategy for all variables was replicated based on the original authors. Yet the low reliability $(\alpha=0.64)$ of the objective knowledge variable could be attributed to how variables were coded ( $0 / 1$ for each correct answer choice, resulting in a knowledge score of 24). Finally, due to the sensitivity of the topic, there may have been a social desirability bias in CHW responses to the survey.

IPV is a complex public health problem that requires intervention at multiple levels to enable victims to receive the care needed to exit abusive relationships. The aforementioned results shed light on the current practice of CHWs in managing clients who are victims of IPV and the need to examine opportunities for additional training and credentialing requirements. Given the dearth of literature that exists on CHWs managing IPV, this research can inform future interventions and state credentialing criteria for CHWs about dealing with clients who are victims of IPV. Due to the unique trust in relationships with their clients, CHWs may serve as promising figures to account for many missed opportunities in addressing IPV. While trust building in CHW relationships with their clients has led to their successful provision of services in areas of maternal and child health and different chronic diseases, some studies have noted inconsistencies in clients seeking help due to the stigma of IPV or CHWs' inability to 
recognize abuse [21]. However, we propose that if given the resources and training to equip them with skills, CHWs may be able to take necessary actions to identify IPV and refer clients to appropriate services. More research is needed to determine specific roles that CHWs can take in addressing IPV, the feasibility and effectiveness of CHWs managing IPV, and how this can be incorporated into credentialing requirements and evaluation criteria of CHWs.

Acknowledgements Support for this research was provided by the Department of Behavioral and Community Health, School of Public Health at the University of Maryland College Park, Dr. Robert S. Gold, and the Asian American Center of Fredrick. We would like to acknowledge Mrs. Tiffany Scott for her assistance in sample recruitment.

Author Contributions The first author designed the study, collected and analyzed the data, and wrote up results. The second author supported the first author in all aspects of the study. The third and fourth authors provided insight on the design, data analysis, and final writeup.

Funding Funding for this research study was provided by the University of Maryland School of Public Health, Department of Behavioral and Community Health, the University of Maryland Graduate School, and the Asian American Center of Frederick.

\section{Declarations}

Conflict of interest The author declare that they have no conflict of interest.

Ethical Approval University of Maryland Institutional Review Board; Maryland Department of Health and Mental Hygiene Institutional Review Board.

Consent to Participate Electronic consent was provided before completing the survey.

\section{References}

1. Breiding, M. J., Basile, K. C., Smith, S. G., Black, M. C., \& Mahendra, R. (2015). Intimate partner violence surveillance: Uniform definitions and recommended data elements version 2.0. National Center for Injury Prevention and Control, Centers for Disease Control and Prevention. https://www.cdc.gov/violencepr evention/pdf/intimatepartnerviolence.pdf

2. Smith, S. G., Zhang, X., Basile, K. C., Merrick, M. T., Wang, J., Kresnow, M., \& Chen, J. (2018). National intimate partner and sexual violence survey (NISVS): 2015 data brief-updated release. Atlanta, GA: National Center for Injury Prevention and Control, Centers for Disease Control and Prevention

3. Fanslow, J., Wise, M. R., \& Marriott, J. (2019). Intimate partner violence and women's reproductive health. Obstetrics, Gynaecology \& Reproductive Medicine, 29(12), 342-350. https://doi.org/ 10.1016/j.ogrm.2019.09.003

4. Breiding, M. J., Black, M. C., \& Ryan, G. W. (2008). Chronic disease and health risk behaviors associated with intimate partner violence-18 U.S. states/territories, 2005. Annals of Epidemiology, 18(7), 538-544. https://doi.org/10.1016/j.annepidem.2008.02.005

5. Plichta, S. B. (2004). Intimate partner violence and physical health consequences: Policy and practice implications. Journal of Interpersonal Violence, 19(11), 1296-1323. https://doi.org/10. 1177/0886260504269685

6. Beydoun, H. A., Beydoun, M. A., Kaufman, J. S., Lo, B., \& Zonderman, A. B. (2012). Intimate partner violence against adult women and its association with major depressive disorder, depressive symptoms and postpartum depression: A systematic review and meta-analysis. Social Science \& Medicine, 75(6), 959-975. https://doi.org/10.1016/j.socscimed.2012.04.025

7. Black, M. C. (2011). Intimate partner violence and adverse health consequences: Implications for clinicians. American Journal of Lifestyle Medicine, 5(5), 428-439. https://doi.org/10.1177/15598 27611410265

8. Karakurt, G., Smith, D., \& Whiting, J. (2014). Impact of intimate partner violence on women's mental health. Journal of Family Violence, 29(7), 693-702. https://doi.org/10.1007/ s10896-014-9633-2

9. Dillon, G., Hussain, R., Loxton, D., \& Rahman, S. (2013). Mental and physical health and intimate partner violence against women: A review of the literature. International Journal of Family Medicine, 313909. https://doi.org/10.1155/2013/313909

10. Campbell, J., Jones, A. S., Dienemann, J., Kub, J., Schollenberger, J., O'Campo, P., Wynne, C. (2002). Intimate partner violence and physical health consequences. Archives of Internal Medicine, 162(10), 1157-1163. https://doi.org/10.1001/archinte.162.10. 1157

11. Bair-Merritt, M. H., Lewis-O'Connor, A., Goel, S., Amato, P., Ismailji, T., Jelley, M. ... Cronholm, P. (2014). Primary carebased interventions for intimate partner violence: A systematic review. American Journal of Preventive Medicine, 46(2), 188194. https://doi.org/10.1016/j.amepre.2013.10.001

12. Chang, J. C., Decker, M., Moracco, K. E., Martin, S. L., Petersen, R., \& Frasier, P. Y. (2003). What happens when health care providers ask about intimate partner violence? A description of consequences from the perspectives of female survivors. Journal of the American Medical Women's Association (1972), 58(2), 76-81

13. McCloskey, L. A., Lichter, E., Williams, C., Gerber, M., Wittenberg, E., \& Ganz, M. (2006). Assessing intimate partner violence in health care settings leads to women's receipt of interventions and improved health. Public Health Reports (Washington, D.C.: 1974), 121(4), 435-444. https://doi.org/10.1177/0033354906 12100412

14. Miller, E., McCaw, B., Humphreys, B. L., \& Mitchell, C. (2015). Integrating intimate partner violence assessment and intervention into healthcare in the united states: A systems approach.Journal of Women's Health, 24(1), 92-99. https://doi.org/10.1089/jwh.2014. 4870

15. U.S. Preventive Services Task Force. (2018). Screening for intimate partner violence, elder abuse, and abuse of vulnerable adults: US preventive services task force final recommendation statement. JAMA, 320(16), 1678-1687. https://doi.org/10.1001/jama.2018. 14741

16. Liebschutz, J., Battaglia, T., Finley, E., \& Averbuch, T. (2008). Disclosing intimate partner violence to health care clinicians-What a difference the setting makes: A qualitative study. BMC Public Health, 8(1), 229. https://doi.org/10.1186/ 1471-2458-8-229.

17. Ambuel, B., Hamberger, L. K., Guse, C. E., Melzer-Lange, M., Phelan, M. B., \& Kistner, A. (2013). Healthcare can change from within: Sustained improvement in the healthcare response to intimate partner violence. Journal of Family Violence, 28(8), 833-847. https://doi.org/10.1007/s10896-013-9550-9.

18. Hamberger, L. K., Rhodes, K., \& Brown, J. (2015). Screening and intervention for intimate partner violence in healthcare settings: Creating sustainable system-level programs. Journal of Women's Health, 24(1), 86-91. https://doi.org/10.1089/jwh.2014.4861. 
19. American Public Health Association. (2000). Community health workers. https://www.apha.org/apha-communities/member-secti ons/community-health-workers

20. Vaughan, K., Kok, M. C., Witter, S., \& Dieleman, M. (2015). Costs and cost-effectiveness of community health workers: Evidence from a literature review. Human Resources for Health, 13(1), 71. https://doi.org/10.1186/s12960-015-0070-y

21. Gatuguta, A., Katusiime, B., Seeley, J., Colombini, M., Mwanzo, I., \& Devries, K. (2017). Should community health workers offer support healthcare services to survivors of sexual violence? A systematic review. BMC International Health and Human Rights, 17(1), 28. https://doi.org/10.1186/s12914-017-0137-z

22. Short, L. M., Alpert, E., Harris, J. M., \& Surprenant, Z. J. (2006). PREMIS: A comprehensive and reliable tool for measuring physician readiness to manage IPV. American Journal of Preventive Medicine, 30(2), 173-180. https://doi.org/10.1016/j.amepre.2005. 10.009 .

23. Bays, A., \& Ingram, K. (2015). Critical synthesis package: Physician readiness to manage intimate partner violence (PREMIS). MedEdPORTAL, 11. https://doi.org/10.15766/mep_2374-8265. 10095

24. DiClemente, C. C., \& Hughes, S. O. (1990). Stages of change profiles in outpatient alcoholism treatment. Journal of Substance Abuse, 2(2), 217-235. https://doi.org/10.1016/s0899-3289(05) 80057-4

25. Prochaska, J. O., \& DiClemente, C. C. (1992). Stages of change in the modification of problem behaviors. Progress in Behavior Modification, 28, 183-218

26. IBM Corp. Released 2020. IBM SPSS statistics for windows, Version 27.0. IBM Corp, Armonk, NY

27. Michalos, A. C. (Ed.). (2014). Kuder-Richardson KR20 Formula. In Encyclopedia of quality of life and well-being research (pp. 3482-3482). Springer, Netherlands. https://doi.org/10.1007/ 978-94-007-0753-5_102199
28. Mukaka, M. (2012). A guide to appropriate use of correlation coefficient in medical research. The Journal of Medical Association of Malawi, 24(3), 69-71.

29. Kaiser, H. F. (1970). A second-generation little jiffy. Psychometrika, 35, 401-415.

30. Office of Disease Prevention and Health Promotion. (n.d.). Reduce intimate partner violence-IVP-DO4. Healthy People 2030. U.S. Department of Health and Human Services. https://health.gov/ healthypeople/objectives-and-data/browse-objectives/violencepr evention/reduce-intimate-partner-violence-ivp-d04

31. Health Resources and Services Administration, Office of Women's Health (2017). The HRSA strategy to address intimate partner violence 2017-2020. Health Resources and Services Administration. https://www.hrsa.gov/sites/default/files/hrsa/HRSA-strategy-intim ate-partnerviolence.pdf

32. Rodgers, M. A., Grisso, J. A., Crits-Christoph, P., \& Rhodes, K. V. (2017). No quick fixes: a mixed methods feasibility study of an urban community health worker outreach program for intimate partner violence. Violence Against Women, 23(3), 287-308. https://doi.org/10.1177/1077801216640383

33. London, K., Carey, M., \& Russell, K. (2016). Community health worker certification requirements by state (pp. 1-10). Connecticut Health Foundation. https://www.cthealth.org/wp-content/uploads/ 2016/02/CHW-Certificaiton-by-State-Final-Final.pdf

Publisher's Note Springer Nature remains neutral with regard to jurisdictional claims in published maps and institutional affiliations. 